\title{
Is It Safe to Move Away from Full Sternotomy for Aortic Valve Replacement?
}

\section{Introduction}

Aortic valve replacement (AVR) is the best long-term treatment for aortic valve disease. There are many surgical approaches to AVR and with the advancement of technology, less invasive treatment modalities are becoming a more popular option for patients. Currently, discussion around minimally invasive approaches are incorporated during the consent process with patients, and in some high-volume centres with experienced surgeons, the minimal access approach has now become a popular option.

Median sternotomy, however, remains the most widely used approach to replace the aortic valve and is associated with a low mortality and morbidity in most centres (1). The current patient population has become older and frailer with more complex comorbidities which has urged the necessity for less invasive approaches to deal with this universal disease of ageing (2). Transcatheter aortic valve implantation (TAVI) is one option in the high-risk surgical group which is now a recognized treatment modality for severe aortic stenosis, aiming to avoid the high surgical burden of a median sternotomy. Some centres are also offering TAVI for intermediate and low risk profile groups, for which the long-term outcomes for patients are still unknown (3). 
Minimally invasive aortic valve replacement (MIAVR) is not novel to the specialty. Cosgrove et al performed the first MIAVR through a parasternal incision in 1996 (4). This approach was followed by AVR via right thoracotomy and an upper hemi-sternotomy $(H S)(5,6)$. Thanks to technological innovations, the instrument set and surgical techniques have moved the field forward gradually.

Minimally invasive surgery (MIS) has gained popularity in the last decade for both patients and surgeons. Within the last decade the expansion of sutureless and rapid deployment valve design and sutured valves using Cor-Knot ${ }^{\circledR}$ have made MIS approaches easier to learn and more convenient with suitability for both low and high-risk patients alike. In order for MIS to become the standard surgical approach the techniques must be reproducible and as safe as surgery via a median sternotomy.

In this article, we present our institutional learning curve and surgical outcomes for both anterior right thoracotomy (ART) and hemi-sternotomy. We aim to demonstrate the safety of moving to MIAVR compared to a median sternotomy (MS).

\section{Patients and Methods:}

Inclusion criteria

All patients who underwent isolated first time AVR between May 2015 and May 2019 were included in the study. The choice of procedure was determined by the operating surgeons' usual practice and whether or not the patient opted for a minimal access approach. Those patients who were offered ART had a computed tomography (CT) angiogram of the entire 
aorta down to the femoral arteries to assess their suitability for an ART approach and safe femoral cannulation.

\section{Exclusion criteria}

All patients who required combination or redo procedures were excluded. Patients with an unsuitable aorta-vascular tree (heavily calcified vessels or femoral arteries less than $6 \mathrm{~mm}$ ) were excluded from the ART approach.

\section{Methods}

All data was extracted retrospectively from our prospectively collected database, we categorized the data by surgical approach into conventional median sternotomy, hemisternotomy and anterior right thoracotomy (ART). Statistical testing was undertaken using Chi-squared test for categorical variables or a one-way Anova with Tukey post-hoc pairwise tests (where appropriate) for continuous variables to explain within which pair(s) the difference exists.

Pre-operative workup

All patients underwent a routine preoperative workup which included: clinical examination, routine bloods, chest X-ray, 12 lead ECG, coronary angiogram when indicated and an anaesthetic review. Patients listed for ART underwent CT angiogram of the aorta with a follow through to the femoral arteries to assess the suitability for ART including peripheral cannulation. Femoral artery size of $7 \mathrm{~mm}$ and above was reassuring for arterial cannulation with a size 21 cannula. Smaller femoral arteries and/or a pressure higher than $300 \mathrm{mmHg}$ in 
the arterial inflow was an indication for bilateral femoral artery cannulation. Aortic calcifications (e.g. porcelain aorta) was also assessed in the CT scan.

\section{Surgical techniques of the MIAVR group}

\section{ART Setup}

\section{Anaesthetic Preparation}

All patients underwent a routine general anaesthetic with a single lumen endotracheal tube; a bronchial blocker to the right main bronchus was inserted to achieve a satisfactory view with right lung deflation. In all MIAVR cases a four-lumen central venous catheter, left radial arterial monitoring line, external defibrillator pads and continuous cerebral oximetry monitoring were used. Transoesophageal echocardiogram (TOE) was applied in all cases and was used to guide cannulation and monitor for retrograde aortic dissection specifically with the ART approach. In our institution ultrasound scanning of the femoral arteries and veins is performed routinely prior to femoral vessels cannulation.

\section{Positioning the Patients}

All patients undergoing sternotomy or hemi-sternotomy were positioned in supine position with a sandbag across both shoulders. In the case of ART, an inflatable bag behind the right hemithorax at the level of the third intercostal space was used to achieve a 30-degree tilt and spread the rib space. 


\section{Cannulation strategy}

In all MS and HS cases, conventional central cannulation techniques were used. Both nonselective and selective antegrade cardioplegia were used. Left ventricle was vented using a right superior pulmonary vein vent. Cross clamping was achieved using a variety of standard cross clamps. In ART cases, peripheral cannulation using the femoral artery and vein was utilized. The side of cannulation was determined according to the preoperative CT scan and intra-operative ultrasound. Femoral cannulation was performed with a cut down using Seldinger technique under TOE guidance. Both body surface area (BSA) and arterial diameter were used to determine the size of Biomedicus, Medtronic cannula selected (size ranges between 19 and 23 French). A multistage venous cannula (Medtronic Bio-medicus ${ }^{\circledR}$ ) was used for venous cannulation. A non-selective aortic root cardioplegia cannula was inserted and a right superior pulmonary vein vent into the left ventricle. A Chitwood clamp inserted thorough the right second intercostal space at the anterior axillary line was used to cross clamp the aorta.

Incision, exposure and closure

In HS cases, we made a J-shaped sternotomy to the level of third or fourth intercostal space. In ART cases, an oblique $5 \mathrm{~cm}$ skin incision on-top of the $3^{\text {rd }}$ rib was performed, followed by blunt dissection of the pectoralis major parallel to the direction of its fibers to the upper border of the $3^{\text {rd }}$ rib. The pleural space was entered after right lung deflation then, the internal mammary vessels were identified, double clipped then divided. The third rib was disarticulated from the sternum using a sternal saw. A soft tissue retractor (Alexis ${ }^{\circledR}$ ) was 
used then gently spread the ribs using a Thoratrack (Medtronic) retractor. The pericardium was opened after commencing cardiopulmonary bypass (CPB).

In both MS and HS, closure was carried out in the standard fashion using stainless steel wires. In ART cases, pericardium was closed after decannulation. Two Blake ${ }^{\circledR}$ chest drains were inserted in the both the pericardium and right pleural space. The third rib was sutured to the sternum with a single number 4-Ethibond suture. The remainder of the incision was closed in layers.

\section{Results}

A total of 661 patients underwent isolated first time AVR between May 2015 and May 2019, of which 429 (65\%) underwent MS, 126 (19\%) had HS and 106 (16\%) had ART. Preoperative characteristics (age, gender, angina class, dyspnoea class, diabetes, smoking history renal failure, pre-operative heart rhythm and ejection fraction) showed no statistical significant difference (see table 1). Unfortunately, the EuroSCORE was not calculated in all patients in our database despite the complete data of its individual components. Hence, we opted not to include it in the preoperative patient characteristics

Intraoperatively, CBP times were significantly higher in all MIAVR groups compared with MS. Further comparative tests showed that the CPB times in the ART group were significantly higher than in the HS group ( $p$ value 0.001). Furthermore, Cross clamp times were also significantly higher in the MIAVR group compared to MS. The HS- versus ART subgroup analysis showed a shorter cross clamp time ( $p$ value 0.004$)$. Valve selection as regards to type (mechanical or biological) was of no statistically significant difference, the data for valve sizes was not complete so was not analyzed. There were $7(6.6 \%)$ conversions in the 
ART group to a full sternotomy including intra-operative conversion and re-exploration for bleeding or tamponade, the conversion data for HS was not recorded accurately and hence unreliable. Potential hemisternotomy conversions were included in the median sternotomy group (table 2).

Postoperatively, there was no mortality within the hospital stay in both HS and ART groups. Seven patients died in the MS group, one patient died of intractable ventricular fibrillation, two patients died of respiratory failure, the others died of, bowel ischaemia, upper gastrointestinal bleeding, post-operative low cardiac output syndrome resulted in multiorgan failure, and after having a large cerebro-vascular event immediately after the surgery. The overall mortality of all patients was $1.06 \%$. There were no new post-operative strokes in the ART group, two patient developed stroke in the HS group and six in the MS group. However, this was not statistically significant across the three groups ( $p$-value 0.70 ). The HS group showed the shortest overall hospital stay whereas MS had the longest hospital stay. The most significant difference in the overall stay was between HS and MS groups ( $p=0.001)$. The ICU stay and postoperative ventilation time was the shortest in the HS group and the longest in the MS group but, this was not significant ( $p$ value 0.26 ). All patients in the ART group were extubated within 24 hours. The postoperative pulmonary complications, gastrointestinal complications and new renal failure were not significant across all groups ( $p$ value $0.40,0.90$ and 0.34 respectively). Blood loss was less in the HS group followed by ART then MS groups. This difference was significant between HS and MS groups ( $p$ value 0.004). The difference between the ART group and HS group was even less significant ( $p$ value 0.571 ) despite trend towards reduced blood loss. Both postoperative blood transfusion and return 
to theatre were not different amongst the three groups ( $p$ value 0.102 and 0.54

respectively). Post-operative arrhythmia had a lower occurrence in the ART group compared to the other two groups. The most significant difference was between the ART group and MS group ( $p$ value 0.004). Furthermore, there were less arrhythmias in HS group compared to MS group $(p=0.031)$. There was a slight tendency towards less arrhythmia in the ART group compared to HS group ( $p$ value 0.28 ) (table 3 ).

\section{Discussion}

Aortic stenosis represents a major health burden in developed countries, especially among the elderly and frail population (7). Aortic valve replacement remains the best long term treatment for severe aortic stenosis and represents a major proportion of adult cardiac surgical practice worldwide (8). Median sternotomy is still the most utilized approach for surgical AVR (SAVR). Despite its physiologic burden, it is a very effective operation associated with low mortality and morbidity with improved symptoms and quality of life as shown in the German registry (1). MIAVR is now becoming more popular for both surgeons and patients. This has resulted in evolution of the surgical techniques of SAVR from MS to the growing use of HS and ART with the ultimate goal to move to totally endoscopic AVR. In this study, we have compared two other approaches for SAVR in comparison to MS. The first approach was HS which has been utilized for longer and the second approach was ART which is less utilized as surgeons are still navigating their learning curves in our institution.

Our results showed no statistically significant difference in between the three groups in terms of mortality, stroke, renal failure and gastrointestinal complications. The intensive care length of stay was not different between three groups however, the total length of 
hospital stay was significantly less in the HS compared to MS group but not between ART and MS or ART and HS. Other studies have demonstrated shorter times in their ART group (9)

Blood loss was significantly less in the HS compared to the MS but not between the other groups. There was no difference in the rate of blood or blood product transfusion. Additionally, the rate of re-exploration for bleeding was similar. Other studies, however, have showed less blood loss and transfusion requirements in their ART patients compared to MS and HS $(10,11)$, Conversion to sternotomy was precisely documented in the ART group, but this was not the case in the HS group. This one of the limitations in this study. A total of five patients in the ART group had a sternotomy when they were re-explored including one patient who was not stable enough to transfer to theatre and was initially re-explored via the ART wound for relief of tamponade. Furthermore, we had two intraoperative conversions due to poor access to the valve. The conversion rate was very different for surgeons and one surgeon stopped offering the ART procedure due to a high conversion rate. This does suggest that the ART approach may not suit all surgeons (12) and regular audit of results is recommended (13).

Interestingly, pulmonary complications were least in the ART group despite the fact that ventilation times were higher, however, the difference was not significant. We expected the ventilation time to be shorter in the MIAVR as was shown by other studies (14-16). One reason for this finding could be due to our departmental policy of requiring a chest X-Ray to ensure lung re-expansion prior to extubation. We are currently implementing an enhanced recovery program for all SAVR patients to ensure shorter ventilation times and hence, shorter ICU stay. Of note, post-operative atrial fibrillation (AF) rates were significantly lower 
in the ART compared to the MS group but not between HS and MS or HS and ART. These findings may reflect the minimal manipulation of the heart in that group of MIAVR as well as the adequacy of myocardial protection. Other studies also report the same outcome (9) Another advantage for MIAVR is that patients experience less post-operative pain; this was demonstrated in a randomized controlled trial by Borger and colleagues and a metanalysis by Brown et al in 2009 (17). Unfortunately, we did not measure pain scores in our patients and hence cannot comment on this. Further studies support early active return to work using the mini sternotomy approach(18). In 2016, Ghanta et al also demonstrated the fiscal advantages associated with less ventilation time, less intensive care (ICU) and total length of hospital stay(HLOS) and fewer blood transfusion requirements (19).

Of note, the heterogeneity in results between different centres applying MIS most likely represents the lack of a structured learning curve and the retrospective nature of the studies with its inherent bias and the small sample size in individual centres (20).

\section{Future Progress}

Many studies have criticized ART procedures over the past decade due to the prolonged CPB and cross clamp times. Although our results do show longer operative times, importantly, this does not translate to more post-operative complications or increased mortality which is supported by other groups in the literature (17). Furthermore, recent studies have shown comparable and sometimes shorter $\mathrm{CPB}$ and cross clamp times to conventional median sternotomy. This discrepancy in results could be attributed to the level of experience of the 
different surgical teams performing the procedure. Naturally, the conclusion is that the shorter the learning curve of the surgeon the better their operative times will be.

Another criticism was the routine use of retrograde CPB in ART procedures which is thought to increase the incidence of stroke. Our results show that there is no statistical difference in stroke rate in all patients. In fact, there was no new stroke incidences in the ART group.

A further criticism was the limited view of the operative field and the need to shift to long shafted instruments. Surgeons, however, with the correct training on equipment adapt well and are able to improve their operative times and results with experience. This ability to adapt to new instruments and a smaller operative field would also facilitate the transition to total endoscopic procedures.

Our study has a few limitations. The retrospective nature of the study which has led to the lack of collection of some relevant data. Euroscore as well as valve sizes data were not complete, we were unable to identify conversions to sternotomy in the HS group. Furthermore, we did not collect echocardiographic outcome data beyond the intraoperative TOE as patients are usually followed up by the cardiologists in their local hospitals. Another limitation was the small number of patients in both HS and ART groups compared to midline sternotomy. These limitations urge the need for a prospective randomized multicentre trial to produce more robust evidence for MIAVR. 


\section{Conclusion}

Based on our experience we believe that it is safe to start a minimally invasive surgical aortic valve replacement program with non-inferior mortality and morbidity to the conventional median sternotomy approach. Hemisternotomy, in our study, has better outcomes than traditional full median sternotomy. A multi-centre randomized control trial may be required to answer the question of universal applicability of minimally invasive surgery. 


\section{References}

1. Holzhey D, Mohr FW, Walther T, Möllmann H, Beckmann A, Kötting J, et al. Current Results of Surgical Aortic Valve Replacement: Insights From the German Aortic Valve Registry. Ann Thorac Surg. 2016;101(2):658-66.

2. Joshi P, Doshi C, Vinchurkar M, Thosani R, Sagar P, Mahajan V. Minimally Invasive Combined Aortic and Mitral Valve Replacement. Heart, Lung and Circulation. 2011;20(4):231-3.

3. Mack MJ, Leon MB, Thourani VH, Makkar R, Kodali SK, Russo M, et al. Transcatheter aorticvalve replacement with a balloon-expandable valve in low-risk patients. $N$ Engl J Med. 2019;380(18):1695-705.

4. Cosgrove DM, Sabik JF. Minimally invasive approach for aortic valve operations. Ann Thorac Surg. 1996;62(2):596-7.

5. Benetti F, Rizzardi JL, Concetti C, Bergese M, Zappetti A. Minimally aortic valve surgery avoiding sternotomy. Eur J Cardiothorac Surg. 1999;16(Supplement_2):S84-S5.

6. Gundry SR, Shattuck OH, Razzouk AJ, del Rio MJ, Sardari FF, Bailey LL. Facile minimally invasive cardiac surgery via ministernotomy. Ann Thorac Surg. 1998;65(4):1100-4.

7. Tavakoli R, Leprince P, Lebreton G, Gassmann M, Jamshidi P, Yamani N, et al. Technique and Patient Selection Criteria of Right Anterior Mini-Thoracotomy for Minimal Access Aortic Valve Replacement. JoVE. 2018(133).

8. Seitz M, Goldblatt J, Paul E, Marcus T, Larobina M, Yap C-H. Minimally Invasive Aortic Valve Replacement Via Right Anterior Mini-Thoracotomy: Propensity Matched Initial Experience. Heart, Lung and Circulation. 2019;28(2):320-6.

9. Krishna RK, Santana O, Mihos CG, Pineda AM, Weiss UK, Lamelas J. Minimally invasive aortic valve replacement in octogenarians performed via a right anterior thoracotomy approach. J Heart Valve Dis. 2014;23(06):671-4. 
10. Santana O, Reyna J, Benjo AM, Lamas GA, Lamelas J. Outcomes of minimally invasive valve surgery in patients with chronic obstructive pulmonary disease. Eur J Cardiothorac Surg. 2012;42(4):648-52.

11. Glauber M, Lio A, Ferrarini M, Miceli A, Montisci A, Donatelli F. Minimally invasive aortic valve replacement with sutureless valves. Indian J Thorac Cardiovasc Surg. 2018;34(2):160-4. 12. Mikus E, Turci S, Calvi S, Ricci M, Dozza L, Del Giglio M. Aortic Valve Replacement Through Right Minithoracotomy: Is it Really Biologically Minimally Invasive? Ann Thorac Surg. 2015;99(3):826-30.

13. Murzi M, Cerillo AG, Gilmanov D, Concistrè G, Farneti $P$, Glauber $M$, et al. Exploring the learning curve for minimally invasive sutureless aortic valve replacement. JTCVS. 2016;152(6):153746.e1.

14. Lamelas J, Sarria A, Santana O, Pineda AM, Lamas GA. Outcomes of Minimally Invasive Valve Surgery Versus Median Sternotomy in Patients Age 75 Years or Greater. Ann Thorac Surg. 2011;91(1):79-84.

15. Santana O, Reyna J, Grana R, Buendia M, Lamas GA, Lamelas J. Outcomes of Minimally Invasive Valve Surgery Versus Standard Sternotomy in Obese Patients Undergoing Isolated Valve Surgery. Ann Thorac Surg. 2011;91(2):406-10.

16. Shehada S-E, Öztürk Ö, Wottke M, Lange R. Propensity score analysis of outcomes following minimal access versus conventional aortic valve replacement. Eur J Cardiothorac Surg. 2015;49(2):464-70.

17. Brown ML, McKellar SH, Sundt TM, Schaff HV. Ministernotomy versus conventional sternotomy for aortic valve replacement: a systematic review and meta-analysis. JTCVS. 2009;137(3):670-9. e5. 
18. Khoshbin E, Prayaga S, Kinsella J, Sutherland F. Mini-sternotomy for aortic valve replacement reduces the length of stay in the cardiac intensive care unit: meta-analysis of randomised controlled trials. BMJ open. 2011;1(2):e000266.

19. Ghanta RK, Lapar DJ, Kern JA, Kron IL, Speir AM, Fonner Jr E, et al. Minimally invasive aortic valve replacement provides equivalent outcomes at reduced cost compared with conventional aortic valve replacement: A real-world multi-institutional analysis. JTCVS. 2015;149(4):1060-5.

20. Murtuza B, De LSR, Rao C, Athanasiou T, Jones C, Darzi A, et al. Minimal Access Aortic Valve Replacement: Is It Worth It? Ann Thorac Surg. 2008;85(3):1121-31. 